PHYSICAL REVIEW D 92, 014501 (2015)

\title{
Study of quark mass dependence of binding energy for light nuclei in $2+1$ flavor lattice QCD
}

\author{
Takeshi Yamazaki, ${ }^{1,2,3}$ Ken-ichi Ishikawa, ${ }^{4,3}$ Yoshinobu Kuramashi, ${ }^{1,2,3}$ and Akira Ukawa ${ }^{3}$ \\ ${ }^{1}$ Faculty of Pure and Applied Sciences, University of Tsukuba, Tsukuba, Ibaraki 305-8571, Japan \\ ${ }^{2}$ Center for Computational Sciences, University of Tsukuba, Tsukuba, Ibaraki 305-8577, Japan \\ ${ }^{3}$ RIKEN Advanced Institute for Computational Science, Kobe, Hyogo 650-0047, Japan \\ ${ }^{4}$ Department of Physics, Hiroshima University, Higashi-Hiroshima, Hiroshima 739-8526, Japan \\ (Received 14 February 2015; revised manuscript received 14 June 2015; published 1 July 2015)
}

\begin{abstract}
We investigate the formation of light nuclei with the nuclear mass number less than or equal to four in $2+1$ flavor QCD using a nonperturbative improved Wilson quark and Iwasaki gauge actions. The quark mass is decreased from our previous work to the one corresponding to the pion mass of $0.30 \mathrm{GeV}$. In each multinucleon channel, the energy shift of the ground state relative to the assembly of free nucleons is calculated on two volumes, whose spatial extents are 4.3 and $5.8 \mathrm{fm}$. From the volume dependence of the energy shift, we distinguish a bound state of multinucleons from an attractive scattering state. We find that all the ground states measured in this calculation are bound states. As in the previous studies at larger $m_{\pi}$, our result indicates that at $m_{\pi}=0.30 \mathrm{GeV}$ the effective interaction between nucleons in the light nuclei is relatively stronger than the one in nature, since the results for the binding energies are larger than the experimental values and a bound state appears in the dineutron channel, which is not observed in experiment. Possible sources of systematic error in this calculation are discussed.
\end{abstract}

DOI: 10.1103/PhysRevD.92.014501

PACS numbers: 11.15.Ha, 12.38.-t, 12.38.Aw, 12.38.Gc

\section{INTRODUCTION}

The strong interaction is the origin of the formation of nuclei. Nonperturbative lattice QCD calculation is a powerful tool to confirm nucleus formation from the first principle of the strong interaction. The nucleus formation was examined in lattice QCD in Ref. [1], in which the binding energies for the ${ }^{4} \mathrm{He}$ and ${ }^{3} \mathrm{He}$ nuclei were calculated in quenched QCD at a heavy quark mass corresponding to the pion mass $m_{\pi}=0.80 \mathrm{GeV}$. In this calculation, a multinucleon bound state was identified by the volume dependence of energy shift of the ground state relative to the assembly of free nucleons. This study was followed by calculations in $N_{f}=3 \mathrm{QCD}$ at $m_{\pi}=0.81 \mathrm{GeV}$ [2] and $2+1$ QCD [3] at $m_{\pi}=0.51 \mathrm{GeV}$. The ${ }^{4} \mathrm{He}$ nucleus formation was also reported in a different approach using the two-nucleon potential calculation in $N_{f}=3$ QCD at $m_{\pi}=0.47,1.02$, and $1.17 \mathrm{GeV}$ [4]. The binding energy reported is much smaller than those in Refs. [1-3]. The authors in Ref. [1] also made the first systematic study of the volume dependence of the energy shift for the twonucleon states in the spin triplet ${ }^{3} S_{1}$ and singlet ${ }^{1} S_{0}$ channels in quenched QCD [5]. This work was extended to the full QCD case in Refs. [2,3,6]; the volume dependence was not examined in the earlier studies of these channels $[7,8]$. The calculation in $N_{f}=2+1 \mathrm{QCD}$ at $m_{\pi}=0.39 \mathrm{GeV}$ [6] was not conclusive of bound state formations in both channels due to large errors of the energy shift. Other calculations $[2,3,5]$, on the other hand, concluded that there is a nucleus in each channel. The latter results conflict with the one from the two-nucleon potential calculation [9].
The results obtained from calculation of the energy shift seem to indicate that the effective interaction among nucleons seems relatively stronger, compared to the kinetic energy of the nucleons, than those in nature. Indeed, in the calculations done to date, the binding energies for the ${ }^{3} \mathrm{He}$ and deuteron are clearly larger than the experimental values; also there is a bound state in the dineutron channel $\left({ }^{1} \mathrm{~S}_{0}\right.$ channel), which has not been observed in nature. A possible explanation of the discrepancy between the lattice QCD results and experiment is the heavier $u, d$ quark masses employed in the calculations. If this is the case, at the physical quark mass the binding energies would agree with those in the nature, and the bound state in the ${ }^{1} \mathrm{~S}_{0}$ channel would disappear. In order to check this scenario, calculations at lighter quark masses than those employed in the previous calculations are necessary. In this paper, we extend our previous calculation [3] at $m_{\pi}=0.51 \mathrm{GeV}$ in $2+1$ flavor QCD to a smaller quark mass of $m_{\pi}=0.30 \mathrm{GeV}$. We investigate whether the light nuclei $\left({ }^{4} \mathrm{He},{ }^{3} \mathrm{He}\right.$, deuteron, and dineutron), which were observed in the previous calculations [1-3,5], are formed or not at this quark mass.

This paper is organized as follows. In Sec. II we explain details of calculation including the simulation parameters for gauge configuration generation and the calculation method for the multinucleon channels. Section III presents the results for the ${ }^{4} \mathrm{He},{ }^{3} \mathrm{He}$, deuteron $\left({ }^{3} \mathrm{~S}_{1}\right)$, and dineutron $\left({ }^{1} \mathrm{~S}_{0}\right)$ channels. Comparisons of our results with those in the previous studies are also discussed. Conclusions and discussions are given in Sec. IV. 


\section{SIMULATION DETAILS}

\section{A. Simulation parameters}

For gauge configuration generation in $2+1$ flavor QCD, we employ the Iwasaki gauge action [10] and a nonperturbative $O(a)$-improved Wilson quark action. The bare coupling is fixed at $\beta=1.90$ for which we use $c_{\mathrm{SW}}=1.715$ [11]. The lattice spacing is $a=0.08995(40) \mathrm{fm}$, corresponding to $a^{-1}=2.194(10) \mathrm{GeV}$, which was determined by $m_{\Omega}=1.6725 \mathrm{GeV}$ [12]. We choose two lattice sizes, $L^{3} \times T=48^{3} \times 48$ and $64^{3} \times 64$, to investigate the spatial volume dependence of the energy shift between the multinucleon ground state and the free nucleons. The physical spatial extents are 4.3 and $5.8 \mathrm{fm}$, respectively. We choose the hopping parameters $\left(\kappa_{u d}, \kappa_{s}\right)=(0.1376314,0.1367299)$ to obtain $m_{\pi}=0.30 \mathrm{GeV}$ and the physical strange quark mass, which are determined by an analysis with the results for $m_{\pi}$ and $m_{s}$ obtained with the same actions but at different hopping parameters $[12,13]$.

We utilize the domain-decomposed hybrid-Monte-Carlo (DDHMC) algorithm [14,15] with mass preconditioning [16], i.e., mass-preconditioned DDHMC (MPDDHMC), for the degenerate light quarks and the UV-filtered polynomial HMC (UVPHMC) algorithm [17] for the strange quark. In both algorithms, we use the Omelyan-MryglodFolk integrator $[18,19]$ in the molecular dynamics evolution. The algorithmic details are given in Ref. [13]. We summarize the simulation parameters in Table I including the block sizes and the preconditioning factor in MPDDHMC and the polynomial order in UVPHMC. We take $\tau=1$ for the trajectory length of the molecular dynamics in all the runs. The step sizes are chosen such that we obtain the reasonable acceptance rates presented in Table I. In the spatial extents of 4.3 and $5.8 \mathrm{fm}, 1000$ and 800 trajectories are generated in four and two streams after thermalization, and the total lengths of trajectory for the measurement are 4000 and 1600, respectively.

We calculate correlation functions in the multinucleon channels in every ten trajectories for both volumes using the same quark action as for the configuration generation. The errors are estimated by jackknife analysis choosing 200 and 160 trajectories for the bin size for the smaller and larger volumes, respectively. Statistics is increased by repeating the measurement of the correlation functions with different source positions on each configuration. We calculate the correlation functions not only in the temporal direction but also in the spatial ones with the use of the space-time rotational symmetry. It allows to increase the statistics by a factor 4 effectively. The parameters of the measurement, e.g., the number of configurations and the bin sizes, are listed in Table II.

\section{B. Calculation method}

We extract the ground state energy in the multinucleon channels and the nucleon mass from the correlation function,

$$
G_{\mathcal{O}}(t)=\langle 0|\mathcal{O}(t) \overline{\mathcal{O}}(0)| 0\rangle,
$$

with $\mathcal{O}$ being proper operators for the ${ }^{4} \mathrm{He},{ }^{3} \mathrm{He},{ }^{3} \mathrm{~S}_{1}$ and ${ }^{1} \mathrm{~S}_{0}$ channels and also the nucleon $N$, which are given in the next subsection.

TABLE I. Simulation parameters for gauge configuration generation at $\left(\kappa_{u d}, \kappa_{s}\right)=(0.1376314,0.1367299)$. The definition of parameters is the same as in Ref. [13].

\begin{tabular}{|c|c|c|c|c|c|c|}
\hline$L^{3} \times T$ & \multicolumn{4}{|c|}{$48^{3} \times 48$} & \multicolumn{2}{|c|}{$64^{3} \times 64$} \\
\hline \# run & \multicolumn{4}{|c|}{4} & \multicolumn{2}{|c|}{2} \\
\hline$\left(N_{0}, N_{1}, N_{2}, N_{3}\right)$ & \multicolumn{4}{|c|}{$(2,2,2,6)$} & \multicolumn{2}{|c|}{$(2,2,2,8)$} \\
\hline Block size & \multicolumn{4}{|c|}{$12^{2} \times 6^{2}$} & \multicolumn{2}{|c|}{$8^{3} \times 4$} \\
\hline$\rho$ & \multicolumn{4}{|c|}{0.998} & \multicolumn{2}{|c|}{0.998} \\
\hline$N_{\text {poly }}$ & \multicolumn{4}{|c|}{320} & \multicolumn{2}{|c|}{340} \\
\hline MD time & 1000 & 1000 & 1000 & 1000 & 800 & 800 \\
\hline$P_{a c c}(\mathrm{HMC})$ & 0.818 & 0.801 & 0.814 & 0.794 & 0.902 & 0.880 \\
\hline$P_{a c c}(\mathrm{GMP})$ & 0.959 & 0.959 & 0.962 & 0.962 & 0.954 & 0.967 \\
\hline
\end{tabular}

TABLE II. Number of configurations, separation of trajectories between each measurement in the units of $\tau$, bin size in jackknife analysis in the units of configuration, number of measurements on each configuration, exponential smearing parameter set $(A, B)$ in Eq. (5), pion mass $m_{\pi}$ and nucleon mass $m_{N}$ are summarized for each lattice size. The number of measurements includes factor 4 by measurement with all four directions, which is explained in the text.

\begin{tabular}{lcccccccc}
\hline \hline$L$ & $T$ & No. configuration & $\tau_{\text {sep }}$ & Bin size & No. measurement & $(A, B)$ & $m_{\pi}[\mathrm{GeV}]$ & $m_{N}[\mathrm{GeV}]$ \\
\hline 48 & 48 & 400 & 10 & 20 & 1152 & $(0.8,0.16)$ & $0.3001(14)$ & $1.057(2)$ \\
64 & 64 & 160 & 10 & 16 & 2048 & $(0.8,0.16)$ & $0.2987(9)$ & $1.053(2)$ \\
\hline \hline
\end{tabular}


We define the energy shift between the multinucleon ground state and free nucleons on finite volume as

$$
\Delta E_{L}=E_{\mathcal{O}}-N_{N} m_{N},
$$

where $E_{\mathcal{O}}$ is the lowest energy level for a multinucleon channel, $N_{N}$ is the number of nucleons in the channel, and $m_{N}$ is the nucleon mass. This quantity is directly extracted from the ratio of the multinucleon correlation function to the $N_{N}$ th power of the nucleon correlation function

$$
R(t)=\frac{G_{\mathcal{O}}(t)}{\left(G_{N}(t)\right)^{N_{N}}},
$$

in the large time region where both correlation functions are dominated by the ground state. We also define an effective energy shift as

$$
\Delta E_{L}^{\mathrm{eff}}=\ln \left(\frac{R(t)}{R(t+1)}\right)
$$

which is utilized to investigate plateau region in the later section. Note that the definition of $\Delta E_{L}$ and $\Delta E_{L}^{\text {eff }}$ follows that in Refs. [3,5], while the sign convention is opposite to that in Ref. [1].

There are two computational difficulties in the calculation of $G_{\mathcal{O}}(t)$ for multinucleon channels. One is a factorially large number of Wick contractions for quarkantiquark fields. To overcome the difficulty, we use the reduction technique of calculation cost proposed in our exploratory work [1]. It is noted that other reduction techniques for the large number of Wick contractions have been proposed for the multimeson [20] and multibaryon [21-23] channels. Another problem is an exponential increase of statistical errors with atomic mass number. For this difficulty, we carry out measurements as much as possible using multiple source points. The number of measurements are a factor 12 and 5 times larger than those in the previous calculation of $m_{\pi}=0.51 \mathrm{GeV}$ [3] for 4.3 and $5.8 \mathrm{fm}$ spatial extents, respectively.

Another difficulty in the nucleus calculation is to distinguish a multinucleon bound state from an attractive scattering state in a finite volume [24-26]. This problem is handled by studying the volume dependence of the measured $\Delta E_{L}$ as in Refs. [1,5]. While the energy shift of an attractive scattering state vanishes in the infinite volume limit as $1 / L^{3}[24,27]$, the physical binding energy of a bound state remains at a finite value in the limit.

\section{Interpolating operators}

The $u, d$ quark propagators are solved with the periodic boundary condition in all of spatial and temporal directions using an exponentially smeared source,

$$
q(\vec{x}, t)=\sum_{\vec{y}} A e^{-B|\vec{x}-\vec{y}|} q_{0}(\vec{y}, t),
$$

for $|\vec{x}| \neq 0$, and $q(\vec{x}, t)=1$ for $|\vec{x}|=0$, after the Coulomb gauge fixing, where $q_{0}$ is the local quark field. We choose the smearing parameters $A=0.8$ and $B=0.16$ on the two volumes to obtain reasonable plateaus of the effective energy for the nucleon and ground states in the multinucleon channels. The stopping condition of the quark propagator $\epsilon=|D x-b| /|b|<10^{-6}$ is applied in both volumes to reduce the calculation time. We have checked in a subset of the configurations that the results with this looser stopping condition agree with the ones using a more stringent stopping condition $\epsilon<10^{-14}$ in more than six digits, even for $G^{4} \mathrm{He}(t)$ at $t=12$. The systematic error coming from the discrepancy is much smaller than the statistical error in the current calculation.

The interpolating operator for the proton is defined as

$$
p_{\alpha}=\varepsilon_{a b c}\left(\left[u_{a}\right]^{t} C \gamma_{5} d_{b}\right) u_{c}^{\alpha},
$$

where $C=\gamma_{4} \gamma_{2}$ and $\alpha$ and $a, b, c$ are the Dirac and color indices, respectively. The neutron operator $n_{\alpha}$ is obtained by replacing $u_{c}^{\alpha}$ by $d_{c}^{\alpha}$ in Eq. (6).

The ${ }^{4} \mathrm{He}$ nucleus has zero total angular momentum and positive parity $J^{P}=0^{+}$with the isospin $I=0$. We employ the simplest ${ }^{4} \mathrm{He}$ interpolating operator with zero orbital angular momentum $L=0$, and hence $J=S$ with $S$ being the total spin [28],

$$
{ }^{4} \mathrm{He}=\frac{1}{\sqrt{2}}(\bar{\chi} \eta-\chi \bar{\eta}),
$$

where

$\chi=\frac{1}{2}([+-+-]+[-+-+]-[+--+]-[-++-])$,

$$
\begin{aligned}
\bar{\chi}= & \frac{1}{\sqrt{12}}([+-+-]+[-+-+]+[+--+]+[-++-] \\
& -2[++--]-2[--++]),
\end{aligned}
$$

with $+/-$ being up/down spin of each nucleon, and $\eta, \bar{\eta}$ are obtained by replacing $+/-$ in $\chi, \bar{\chi}$ by $p / n$ for the isospin.

The ${ }^{3} \mathrm{He}$ nucleus has $J^{P}=\frac{1}{2}+, I=\frac{1}{2}$ and $I_{z}=\frac{1}{2}$. We employ the interpolating operator in Ref. [29],

$$
\begin{aligned}
{ }^{3} \mathrm{He}= & \frac{1}{\sqrt{6}}\left(\left|p_{-} n_{+} p_{+}\right\rangle-\left|p_{+} n_{+} p_{-}\right\rangle+\left|n_{+} p_{+} p_{-}\right\rangle\right. \\
& \left.-\left|n_{+} p_{-} p_{+}\right\rangle+\left|p_{+} p_{-} n_{+}\right\rangle-\left|p_{-} p_{+} n_{+}\right\rangle\right) .
\end{aligned}
$$

The two-nucleon operators for the ${ }^{3} \mathrm{~S}_{1}$ and ${ }^{1} \mathrm{~S}_{0}$ channels are given by 


$$
\begin{aligned}
& N N^{{ }^{3} \mathrm{~S}_{1}}{ }_{1}(t)=\frac{1}{\sqrt{2}}\left[p_{+}(t) n_{+}(t)-n_{+}(t) p_{+}(t)\right], \\
& N N_{{ }^{1} \mathrm{~S}_{0}}(t)=\frac{1}{\sqrt{2}}\left[p_{+}(t) p_{-}(t)-p_{-}(t) p_{+}(t)\right],
\end{aligned}
$$

respectively. In the ${ }^{3} S_{1}$ channel the operators for the other two spin components are constructed in a similar way. We increase statistics by averaging over the three correlation function with each spin component operator.

Using the interpolating operators above, we calculate correlation functions in each channel. For the source operators in all correlation functions, we insert the smeared quark fields of Eq. (5) for each nucleon operator located at the same spatial point $\vec{x}$. Each nucleon in the sink operator, on the other hand, is composed of the point quark fields corresponding to $q_{0}$ in Eq. (5), and projected to zero spatial momentum. To save the computational cost we use the nonrelativistic quark operators, in which the Dirac index in Eq. (6) is restricted to the upper two components in the Dirac representation.

\section{RESULTS}

\section{A. Nucleon and pion masses}

The results for effective $m_{N}$ in the two volumes are shown in Fig. 1 together with the exponential fit result of $C_{N}(t)$ and the one standard deviation error band. Plateaus are clearly seen for $t \geq 8$ for both volumes. The difference of the fit results between the two volumes is 1.4 standard deviations, and hence statistically not very significant. We also do not expect much finite size effect for these large volumes satisfying $m_{\pi} L>6$. In the following sections, we therefore consider that the difference is caused by statistics, and will not estimate the systematic error from it. The pion

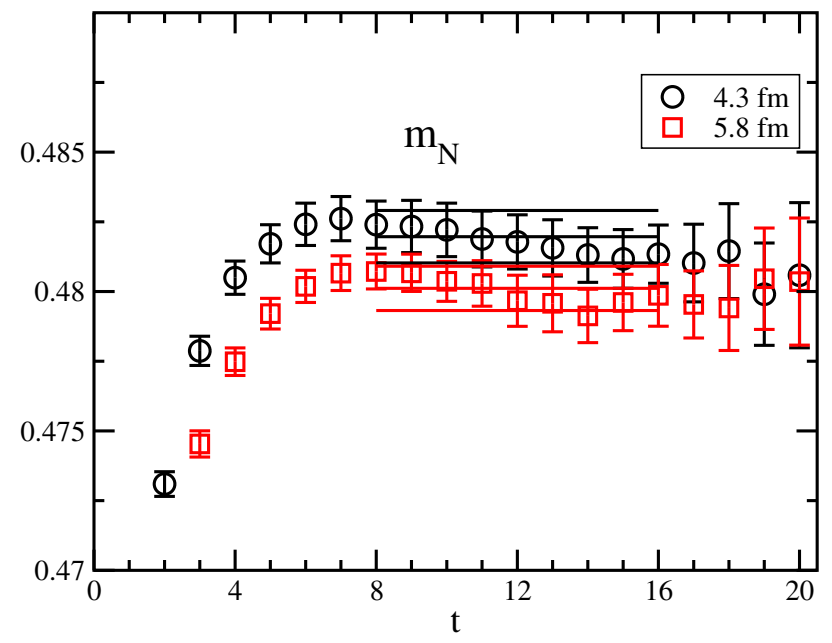

FIG. 1 (color online). Nucleon effective masses on $(4.3 \mathrm{fm})^{3}$ and $(5.8 \mathrm{fm})^{3}$ volumes in lattice unites. The fit result with one standard deviation error band is expressed by solid lines.

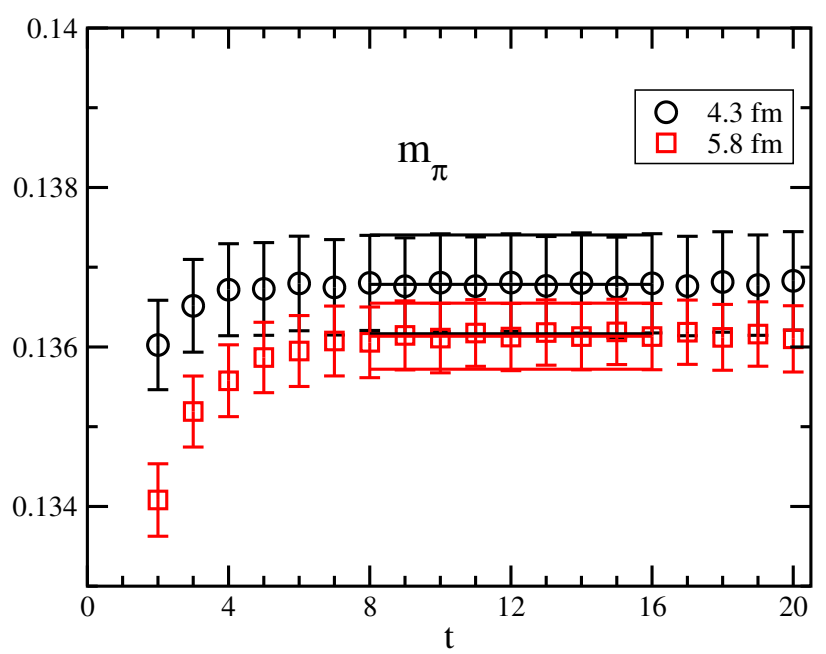

FIG. 2 (color online). Same as Fig. 1, but for pion effective masses.

effective masses in each volume show better consistency than the nucleon mass, as presented in Fig. 2. Those fit results are tabulated in Table II.

\section{B. ${ }^{4}$ He channel}

The effective energy shift $\Delta E_{L}^{\text {eff }}$ defined in Eq. (4) is shown in Fig. 3 for the two volumes. Clear signals are seen for $t \leq 10$, but for larger $t$ the statistical error increases rapidly. A plateau appears at $t=9-12$ on the larger volume, while it is not clearly seen on the smaller volume. More statistics is desirable for establishing a plateau in this case. We calculate the energy shift $\Delta E_{L}$ in Eq. (2) by a single exponential fit of $R(t)$ in Eq. (3) using the same range $t=9-12$ for the two volumes. The systematic error is estimated from the variation of the fit results with six different fit ranges, where the minimum or maximum time slice is changed by \pm 1 , and the minimum and maximum time slices are changed by +1 and +2 . The central fit result is shown in Fig. 3 by solid lines with the band representing the statistical error. The dashed lines denote the total error adding the statistical and systematic errors by quadrature. In Fig. 4 we illustrate how we estimate the systematic error. Shown in the figure are the results of eight fits obtained by shifting the fitting range as explained in the figure caption. The horizontal band with solid lines shows the total error obtained by adding the statistical error and the systematic error from the seven fits on the left by quadrature. We observe that the seven fits on the left reasonably covers the variation, with the eighth fit at the rightmost with the fitting range shifted by +3 from $\left(t_{\min }, t_{\max }\right)$ falling within the band of solid lines within one sigma. We therefore consider that our estimate of systematic errors is reasonable under the current statistics. The values of $\Delta E_{L}$ with the statistical and systematic errors are summarized in Table III.

Figure 5 shows the volume dependence of $\Delta E_{L}$ as a function of $1 / L^{3}$. The inner bar of each data denotes the 

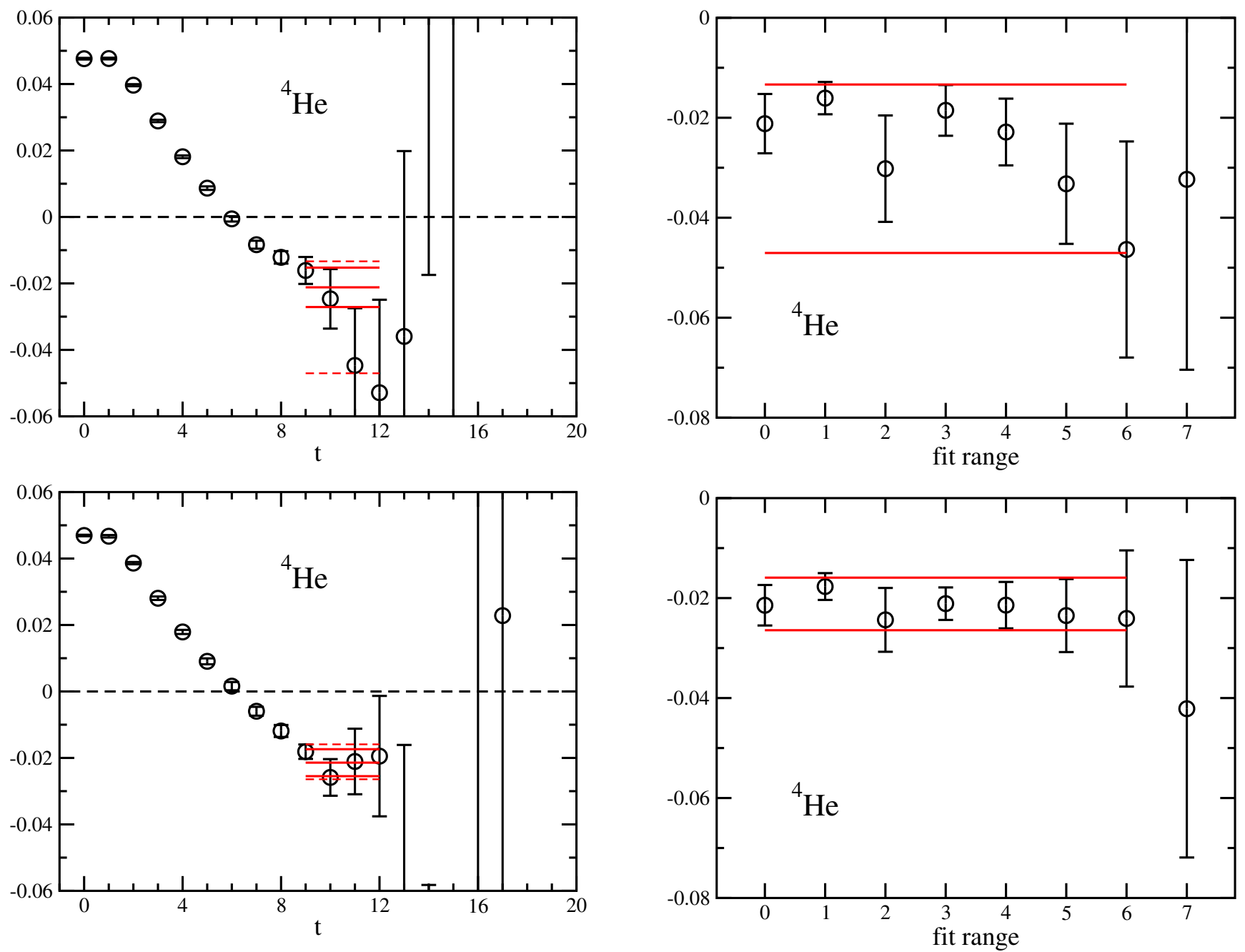

FIG. 3 (color online). Effective energy shift $\Delta E_{L}^{\text {eff }}$ for ${ }^{4} \mathrm{He}$ channel on $(4.3 \mathrm{fm})^{3}$ (top) and $(5.8 \mathrm{fm})^{3}$ (bottom) volumes in lattice units. The fit result with one standard deviation error band and total error including the systematic one is expressed by solid and dashed lines, respectively.

statistical error and the outer bar represents the total error with the statistical and systematic ones added in quadrature. Since the volume dependence is not large, we estimate the energy shift in the infinite volume limit $\Delta E_{\infty}$ by a constant fit as presented by solid line and open circle in Fig. 5. An exponential type extrapolation, $\Delta E_{L}=\Delta E_{\infty}+$ $C \exp \left(-C_{e} L\right)$, cannot be carried out in this paper due to the smaller number of data than its free parameters. The systematic error is estimated from the variation of the central values obtained by 49 fits. The 49 fits are constant fits with a various combination of $7^{2}$ data set, where in each volume we have seven data with different fit range of $R(t)$ as explained in the above. The result of $\Delta E_{\infty}$ with the statistical and systematic errors are tabulated in Table III. From the result that $\Delta E_{\infty}$ is nonzero and negative, we conclude that the ground state is bound in this channel. The binding energy equals $-\Delta E_{\infty}=47(7)\left(\begin{array}{c}+20 \\ -11\end{array}\right) \mathrm{MeV}$ where

FIG. 4 (color online). The fit range dependence of energy shift $\Delta E_{L}$ for ${ }^{4} \mathrm{He}$ channel on $(4.3 \mathrm{fm})^{3}$ (top) and $(5.8 \mathrm{fm})^{3}$ (bottom) volumes in lattice units. The horizontal axis corresponds to the fit range $\left(t_{\min }, t_{\max }\right),\left(t_{\min }-1, t_{\max }\right),\left(t_{\min }+1, t_{\max }\right),\left(t_{\min }, t_{\max }-1\right)$, $\left(t_{\min }, t_{\max }+1\right),\left(t_{\min }+1, t_{\max }+1\right),\left(t_{\min }+2, t_{\max }+2\right)$, and $\left(t_{\min }+3\right.$, $\left.t_{\max }+3\right)$ from left to right. $t_{\min }$ and $t_{\max }$ are minimum and maximum time slices of the fit range, respectively, whose values are explained in the text. The total error band including the statistical and systematic is expressed by solid lines.

the first and second errors are statistical and systematic, respectively.

The result for $-\Delta E_{\infty}$ is compared with the experimental value of $28.3 \mathrm{MeV}$ and with the previous three results [1-3] in Fig. 6. The binding energy for $m_{\pi}=0.3 \mathrm{GeV}$ obtained in this paper is similar in magnitude with our previous results for $N_{f}=2+1$ at $m_{\pi}=0.51 \mathrm{GeV}$ [3] and $N_{f}=0$ at $m_{\pi}=0.80 \mathrm{GeV}$ [5]. Compared to experiment, if one used the upper total error, our current value is consistent within $1.5 \sigma$. The result of the $N_{f}=3$ calculation at $m_{\pi}=$ $0.81 \mathrm{GeV}[2]$ is about 3 times larger than the other results. This difference might be due to different quark masses of the calculation or dynamical quark effects. On the other 
TABLE III. Energy shift $-\Delta E_{L}$ in physical units and fit range for ${ }^{4} \mathrm{He}$ and ${ }^{3} \mathrm{He}$ channels on each spatial volume. Extrapolated results in the infinite spatial volume limit are also presented. The first and second errors are statistical and systematic, respectively.

\begin{tabular}{lccccc}
\hline \hline & \multicolumn{2}{c}{${ }^{4} \mathrm{He}$} & & \multicolumn{2}{c}{${ }^{3} \mathrm{He}$} \\
\cline { 2 - 3 } \cline { 5 - 6 } & $-\Delta E_{L}[\mathrm{MeV}]$ & Fit range & & $-\Delta E_{L}[\mathrm{MeV}]$ & Fit range \\
\hline 48 & $46(13)\left({ }_{-11}^{+55}\right)$ & $9-12$ & & $18.7(1.9)\left({ }_{-3.1}^{+15}\right)$ & $8-12$ \\
64 & $47(9)\left({ }_{-8}^{+6}\right)$ & $9-12$ & & $23.7(1.6)\left({ }_{-2.5}^{+13}\right)$ & $9-12$ \\
$\infty$ & $47(7)\left({ }_{-11}^{+20}\right)$ & $\ldots$ & & $21.7(1.2)\left({ }_{-1.6}^{+13}\right)$ & $\cdots$ \\
\hline \hline
\end{tabular}

hand, the result obtained with the two-nucleon potential extracted from $N_{f}=3$ calculations at $m_{\pi}=0.47 \mathrm{GeV}$ [4] has a very small binding energy, $\Delta E=5.1 \mathrm{MeV}$, compared to the other results.

\section{C. ${ }^{3} \mathrm{He}$ channel}

Figure 7 shows the effective energy shift $\Delta E_{L}^{\text {eff }}$ in Eq. (4) for the two volumes. The signals are better than those in the ${ }^{4} \mathrm{He}$ channel shown in Fig. 3. A plateau is seen for the smaller volume case, while it is less clear in the region of $t=8-12$ for the larger volume case. The energy shift $\Delta E_{L}$ in Eq. (2) is determined by an exponential fit to $R(t)$ in Eq. (3) with the fit range of $t=8-12$ and 9-12 for the smaller and larger volumes, respectively. The systematic error of $\Delta E_{L}$ is estimated in the same way as for the ${ }^{4} \mathrm{He}$ case as described in the above subsection. The fit results with the statistical and systematic errors are shown in Fig. 7 and Table III. The explanations for the solid and dashed lines are given in the previous subsection. Figure 8 shows how we estimate the systematic error. The relative

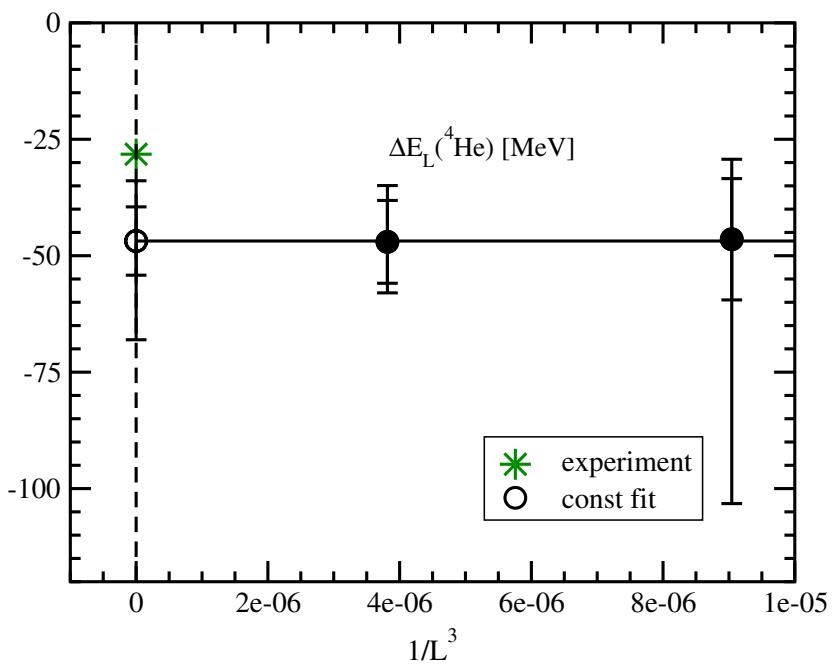

FIG. 5 (color online). Spatial volume dependence of $\Delta E_{L}$ for ${ }^{4} \mathrm{He}$ channel in $\mathrm{MeV}$ units. The outer bar denotes the total error of statistical and systematic ones added in quadrature. The inner bar is for the statistical error. The constant fit result is shown by an open circle symbol. The experimental value (star) is also presented.

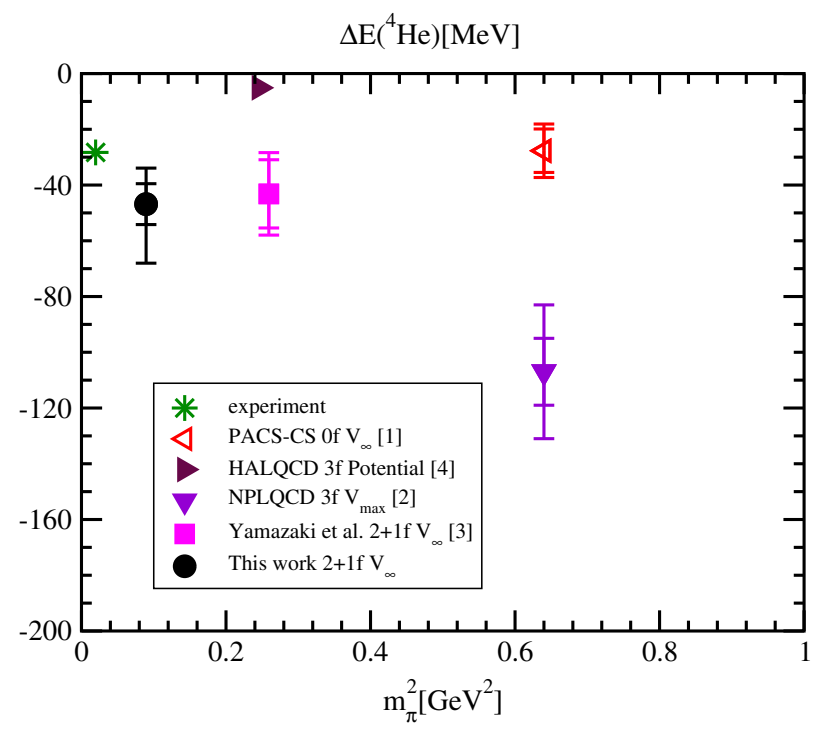

FIG. 6 (color online). $\quad m_{\pi}^{2}$ dependence of energy shift for ${ }^{4} \mathrm{He}$ channel in $\mathrm{MeV}$ units. Open and closed symbols denote the quenched [1] and full QCD [2-4] results, respectively. The results of Refs. [1,3] and this paper are the ones in the infinite volume limit. The error of the result obtained from the two-nucleon potential was not estimated in Ref. [4]. The experimental result (star) is also presented for comparison.

difference of the extra fit result with the slid fit range by +3 , as explained in the previous subsection, from the central fit result with the total error is less than $1.6 \sigma$.

A weak volume dependence of $\Delta E_{L}$ observed in Fig. 9 is similar to those in the previous results [1-3]. A constant fit of $\Delta E_{L}$ with only the statistical error gives a large value of $\chi^{2} / \mathrm{DOF}=4.1$, where DOF denotes degrees of freedom. It agrees with the two data within the total error as shown in Fig. 9, however. Thus, we take the constant fit result as the estimate of the central value of $\Delta E_{\infty}$ in this calculation.

We estimate the systematic error of $\Delta E_{\infty}$ in the same way to the ${ }^{4} \mathrm{He}$ case. We omit 18 constant fit results, however, with $\chi^{2} / \mathrm{DOF}>4.1$. The extrapolated result of $\Delta E_{\infty}$ is clearly nonzero and negative as presented in Fig. 9. Thus the ground state is a bound state, corresponding to the ${ }^{3} \mathrm{He}$ nucleus, with a binding energy of $-\Delta E_{\infty}=$ 21.7 $(1.2)\left({ }_{-1.6}^{+13}\right) \mathrm{MeV}$, where the first and second errors are statistical and systematic, respectively.

The quark mass dependence of the energy shift is plotted in Fig. 10. Our present result together with our two previous calculations $[1,3]$ show very small dependence, while NPLQCD reported a much deeper bound state [2]. All lattice results in the figure have the binding energy larger than the experimental value $7.72 \mathrm{MeV}$.

\section{Two-nucleon channels}

We present $\Delta E_{L}^{\text {eff }}$ in Eq. (4) for the ${ }^{3} S_{1}$ and ${ }^{1} S_{0}$ channels in Figs. 11 and 12, respectively. The signals are clean up to $t \approx 14$, but statistical fluctuations spoil the signals in the 

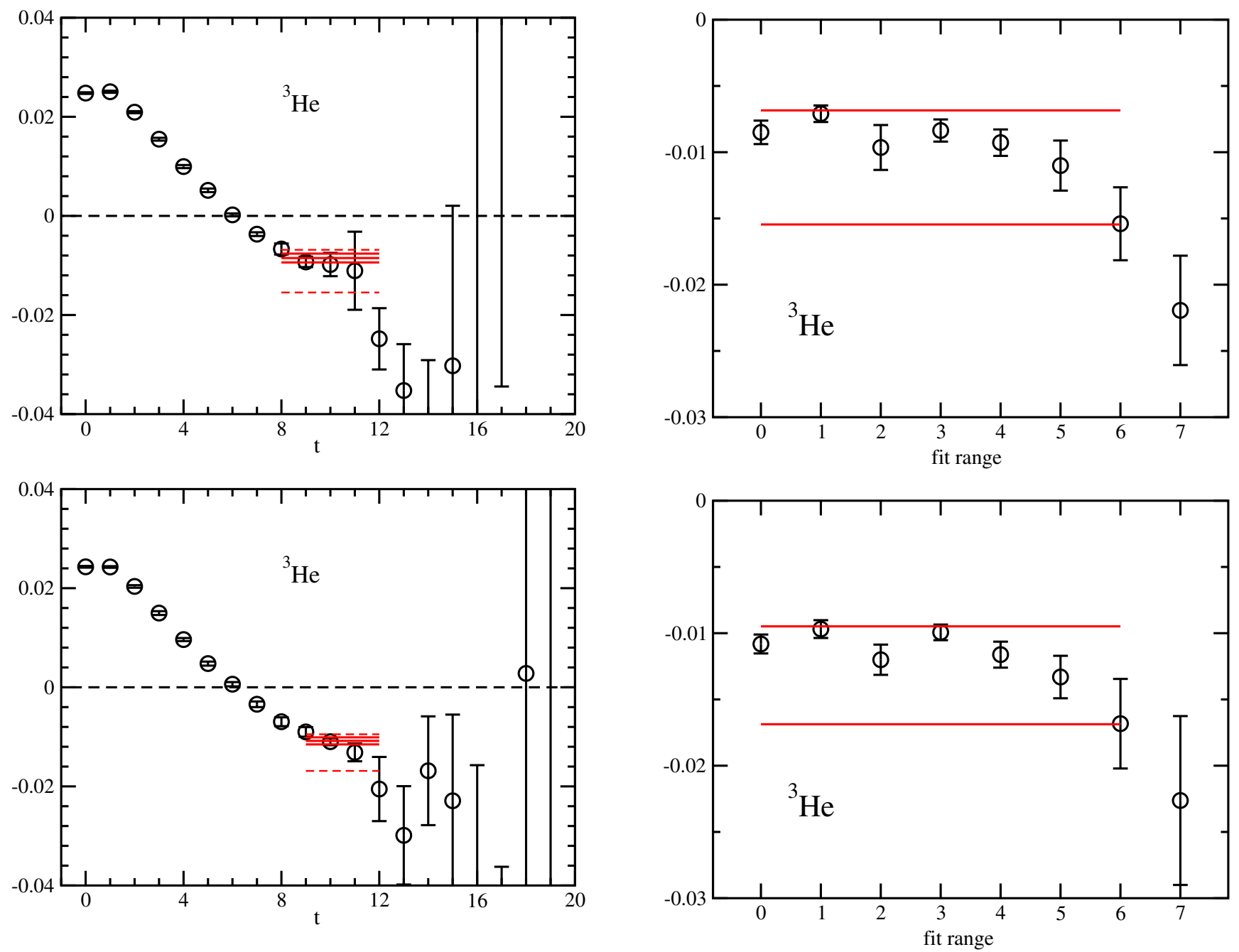

FIG. 7 (color online). Same as Fig. 3, but for the ${ }^{3}$ He channel.

FIG. 8 (color online). Same as Fig. 4 , but for the ${ }^{3} \mathrm{He}$ channel.

larger time region. The values of $\left|\Delta E_{L}^{\text {eff }}\right|$ in the ${ }^{1} \mathrm{~S}_{0}$ channel are smaller than those in the ${ }^{3} S_{1}$ channel. A similar trend was seen in the previous studies $[2,3,5]$. We observe a clear plateau with a negative energy shift for $9 \lesssim t \lesssim 14$, although of a less quality for the ${ }^{3} S_{1}$ channel for the smaller volume. We determine $\Delta E_{L}$ by an exponential fit to $R(t)$ of Eq. (3) with the fixed fit range of $t=9-13$ for the ${ }^{3} \mathrm{~S}_{1}$ channel, and with $t=10-14$ for ${ }^{1} \mathrm{~S}_{0}$. The fit results are presented in Figs. 11 and 12, and are summarized in Table IV. The systematic error estimations for the ${ }^{3} S_{1}$ and ${ }^{1} \mathrm{~S}_{0}$ channels using the results with the several fit ranges, as in the ${ }^{4} \mathrm{He}$ and ${ }^{3} \mathrm{He}$ cases, are presented in Figs. 13 and 14, respectively.

The volume dependence of the energy shift in the two channels is shown in Figs. 15 and 16. In both channels, the volume dependences are mild, so that the data can be reasonably fitted by a constant. The results for the constant fit are nonzero and negative. This indicates that the ground states in the two channels are bound states. The same conclusion is also obtained by a fit including finite volume effects of the two-particle bound state $[25,26]$,

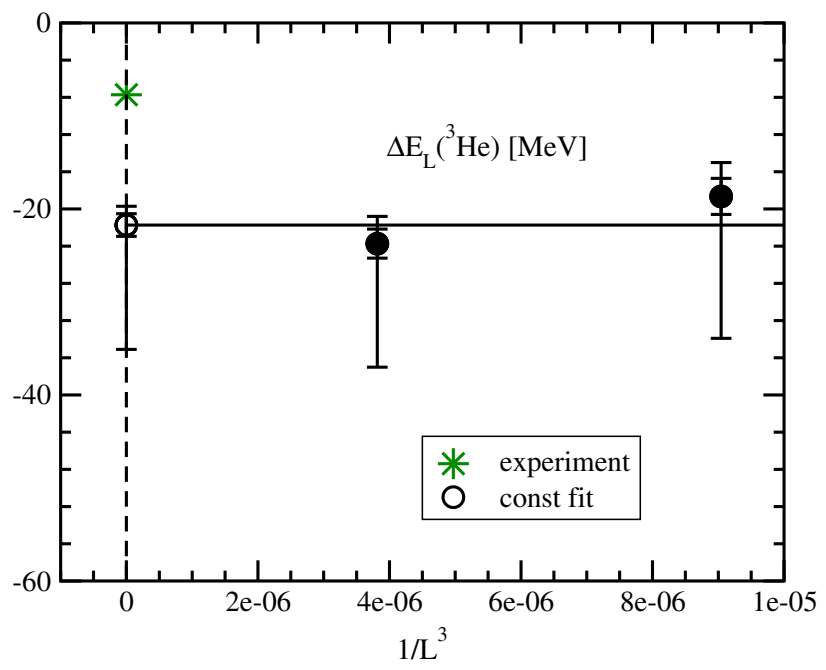

FIG. 9 (color online). Same as Fig. 5, but for the ${ }^{3} \mathrm{He}$ channel. 


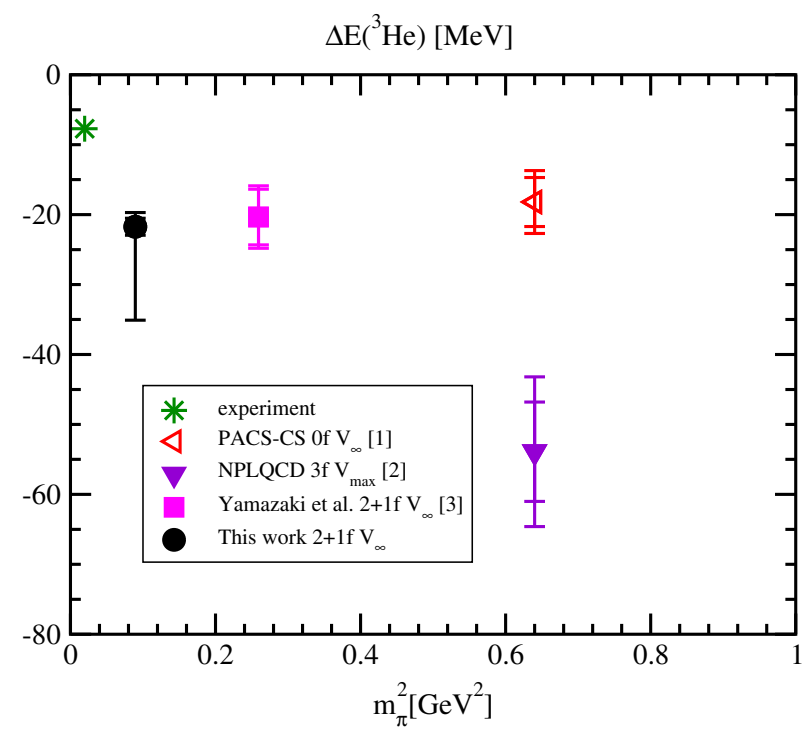

FIG. 10 (color online). Same as Fig. 6, but for the ${ }^{3} \mathrm{He}$ channel.
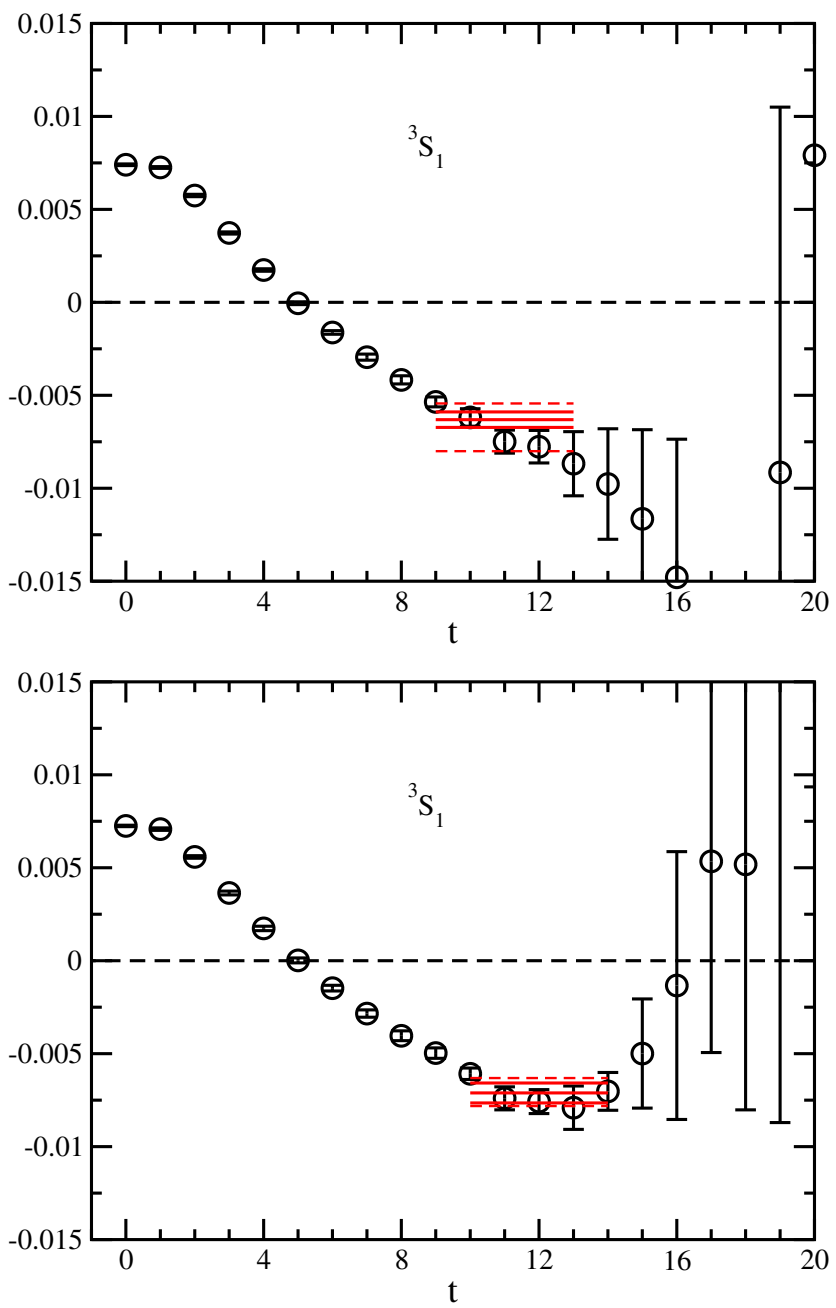

FIG. 11 (color online). Same as Fig. 3, but for the ${ }^{3} \mathrm{~S}_{1} N N$ channel.
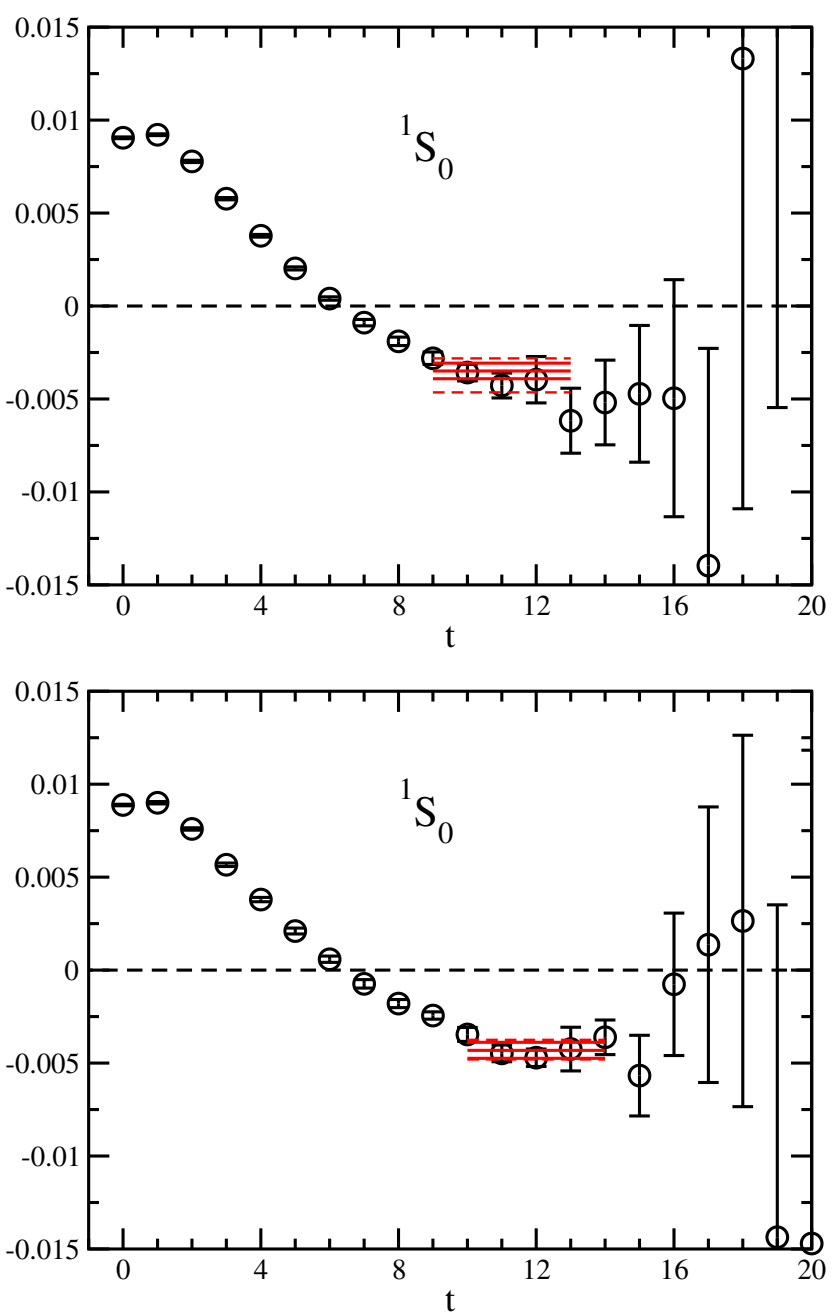

FIG. 12 (color online). Same as Fig. 3, but for the ${ }^{1} \mathrm{~S}_{0} N N$ channel.

$$
\Delta E_{L}=-\frac{\gamma^{2}}{m_{N}}\left\{1+\frac{C_{\gamma}}{\gamma L} \sum_{\vec{n}}^{\prime} \frac{\exp \left(-\gamma L \sqrt{\vec{n}^{2}}\right)}{\sqrt{\vec{n}^{2}}}\right\}
$$

where $\gamma$ and $C_{\gamma}$ are free parameters, $\vec{n}$ is a three-dimensional integer vector, and $\sum_{\vec{n}}^{\prime}$ denotes the summation without $|\vec{n}|=0$. In the fit, we use the weighted average value of $m_{N}$ with the two volume data. In the above equation, it is assumed that

TABLE IV. Same as Table III for ${ }^{3} S_{1}$ and ${ }^{1} S_{0}$ channels.

\begin{tabular}{cccccc}
\hline \hline & \multicolumn{2}{c}{${ }^{3} \mathrm{~S}_{1}$} & & \multicolumn{2}{c}{${ }^{1} \mathrm{~S}_{0}$} \\
\cline { 2 - 3 } & $-\Delta E_{L}[\mathrm{MeV}]$ & Fit range & & $-\Delta E_{L}[\mathrm{MeV}]$ & Fit range \\
\hline 48 & $13.8(0.9)\left({ }_{-1.7}^{+3.6}\right)$ & $9-13$ & & $7.7(0.9)\left({ }_{-1.2}^{+2.4}\right)$ & $9-13$ \\
64 & $15.6(1.2)\left({ }_{-1.3}^{+1.0}\right)$ & $10-14$ & $9.5(0.9)\left({ }_{-0.8}^{+0.5}\right)$ & $10-14$ \\
$\infty$ & $14.5(0.7)\left({ }_{-0.8}^{+2.4}\right)$ & $\ldots$ & $8.5(0.7)\left({ }_{-0.5}^{+1.6}\right)$ & $\ldots$ \\
\hline \hline
\end{tabular}



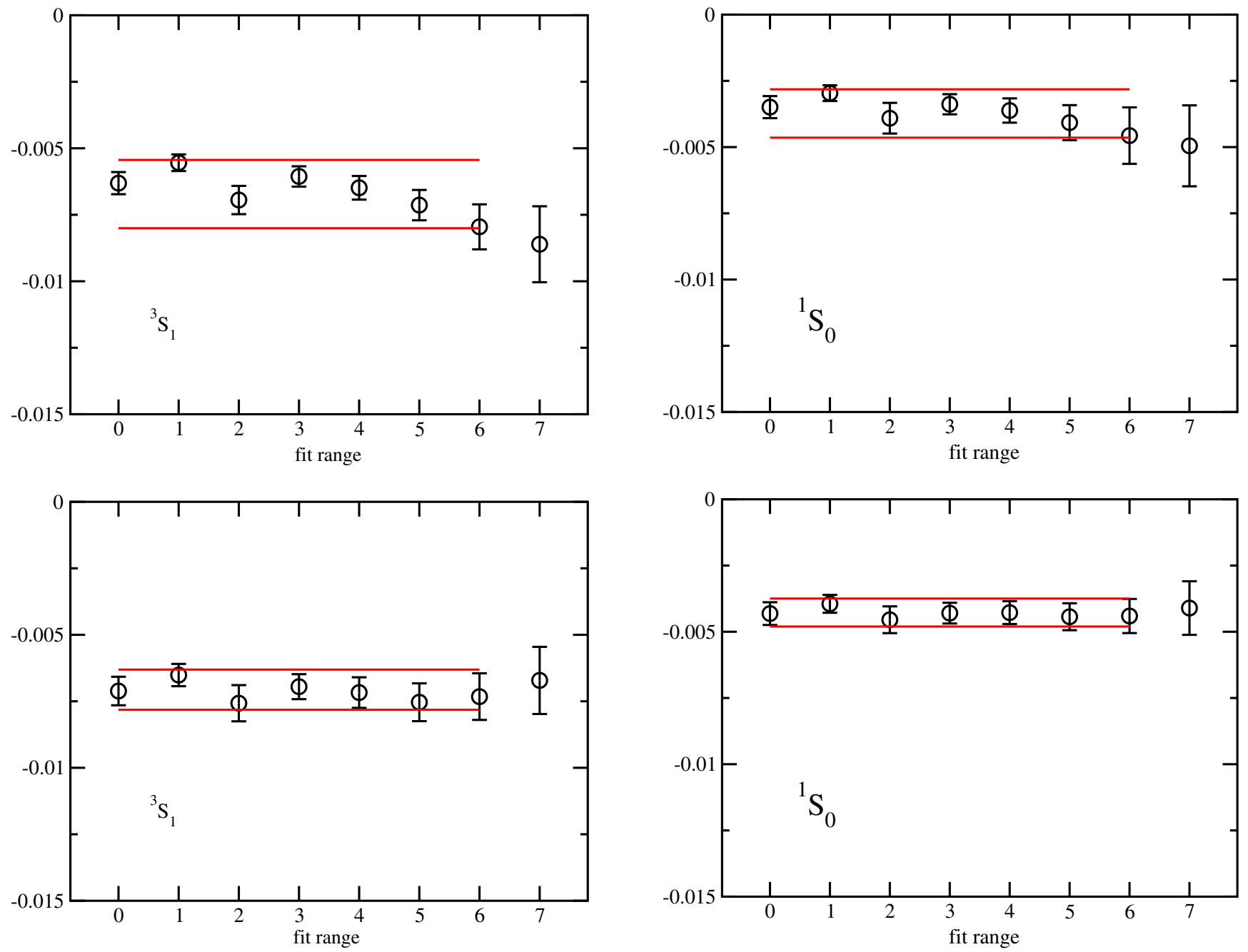

FIG. 13 (color online). Same as Fig. 4, but for the ${ }^{3} \mathrm{~S}_{1} N N$ channel.

$$
-\Delta E_{\infty}=\frac{\gamma^{2}}{m_{N}} \approx 2 m_{N}-2 \sqrt{m_{N}^{2}-\gamma^{2}}
$$

Note that the degrees of freedom are zero in the fit with Eq. (13). The fit result is presented in each figure at $1 / L^{3}=0$. We take the constant fit as the central value of the binding energy $-\Delta E_{\infty}$, and estimate the systematic error in the same way as in other channels. In the systematic error estimation, we include the fit result using Eq. (13), while we exclude nine and eight constant fit results in the ${ }^{3} \mathrm{~S}_{1}$ and ${ }^{1} \mathrm{~S}_{0}$ channels, respectively, which yield $\chi^{2} / \mathrm{DOF}>3$. The results for the binding energy are $-\Delta E_{\infty}=14.5(0.7)\left({ }_{-0.8}^{+2.4}\right) \mathrm{MeV}$ for the ${ }^{3} \mathrm{~S}_{1}$ channel and $8.5(0.7)\left(\begin{array}{l}+1.6 \\ -0.5\end{array}\right) \mathrm{MeV}$ for ${ }^{1} \mathrm{~S}_{0}$ with the first and second errors being the statistical and systematic, which are also summarized in Table IV.

In Figs. 17 and 18, the results for $\Delta E_{\infty}$ in the present paper are compared with those of the previous studies [2,3,5-8] as a function of $m_{\pi}^{2}$. Almost all results report

FIG. 14 (color online). Same as Fig. 4, but for the ${ }^{1} \mathrm{~S}_{0} N N$ channel.

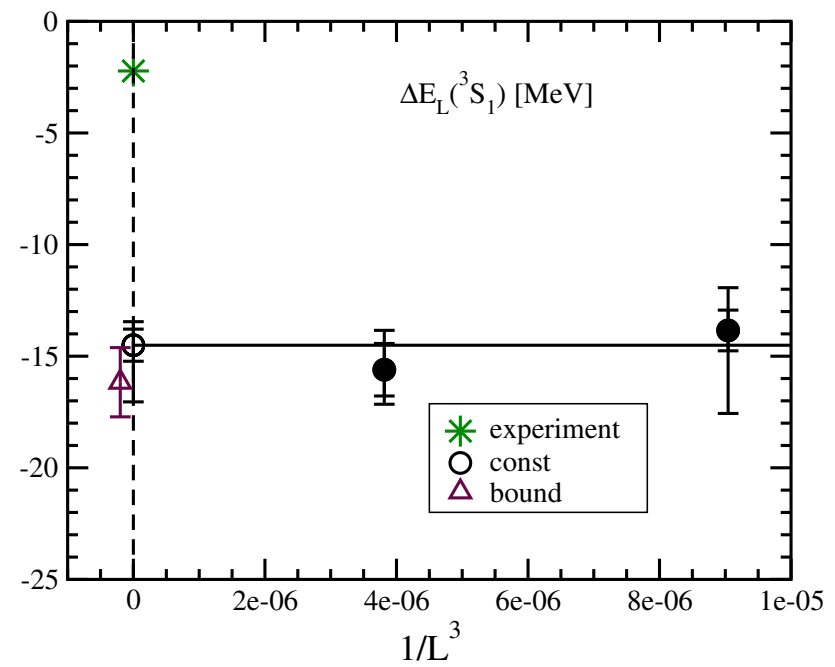

FIG. 15 (color online). Same as Fig. 5, but for the ${ }^{3} \mathrm{~S}_{1} N N$ channel. The fit result using finite volume dependence of two-particle binding energy Eq. (13) is also plotted. 


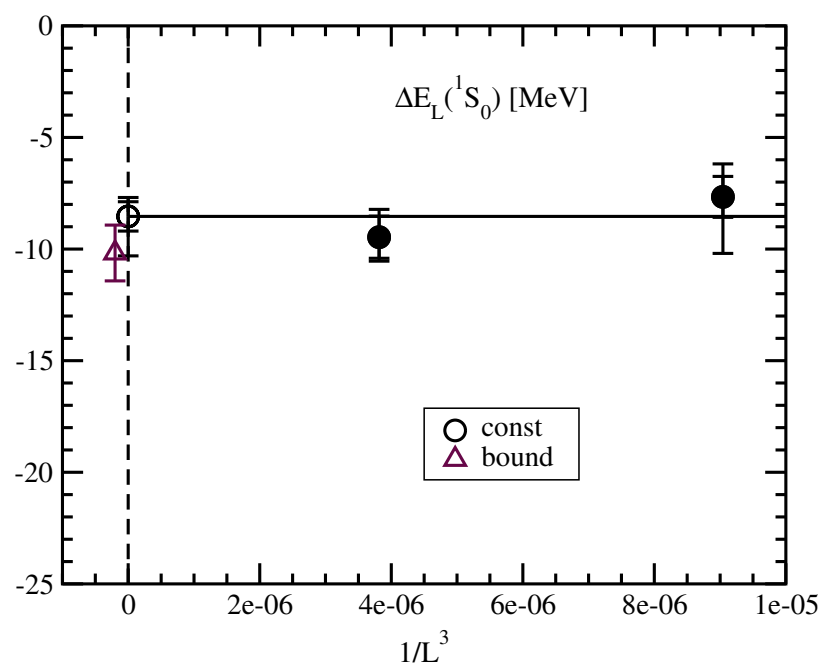

FIG. 16 (color online). Same as Fig. 15, but for the ${ }^{1} \mathrm{~S}_{0} N N$ channel.

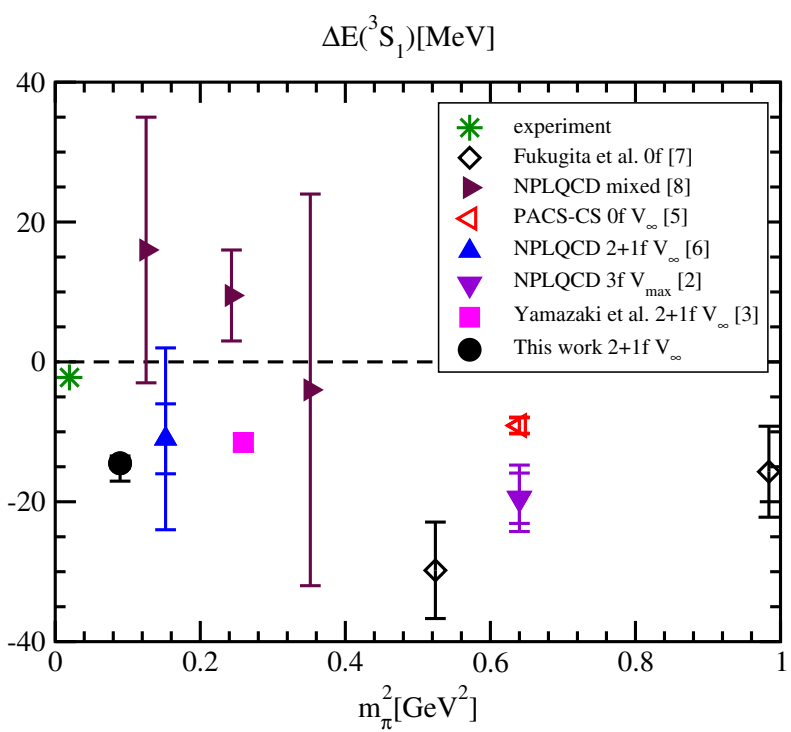

FIG. 17 (color online). Same as Fig. 6, but for the ${ }^{3} \mathrm{~S}_{1} N N$ channel. Open and closed symbols denote the quenched [5,7] and full QCD [2,3,6,8] results, respectively. The results of Refs. $[2,3,5,6]$ and this paper are the ones in the infinite volume limit.

negative values, except for those of Ref. [8] with large errors. The earlier calculations $[7,8]$ did not investigate the volume dependence of $\Delta E_{L}$. More recent studies [2,3,5,6] examined the dependence and estimated the infinite volume value through extrapolations $[3,5,6]$ or checked that there is no significant volume dependence of $\Delta E_{L}$ [2]. All the recent results suggest that the ground states in both channels are bound states. One exception is Ref. [6] where the conclusion is not clear due to large errors.

While lattice results are mutually qualitatively consistent, they differ from experiment in more than one aspects. For the ${ }^{3} \mathrm{~S}_{1}$ channel, the binding energy $-\Delta E_{\infty}$ found in the lattice

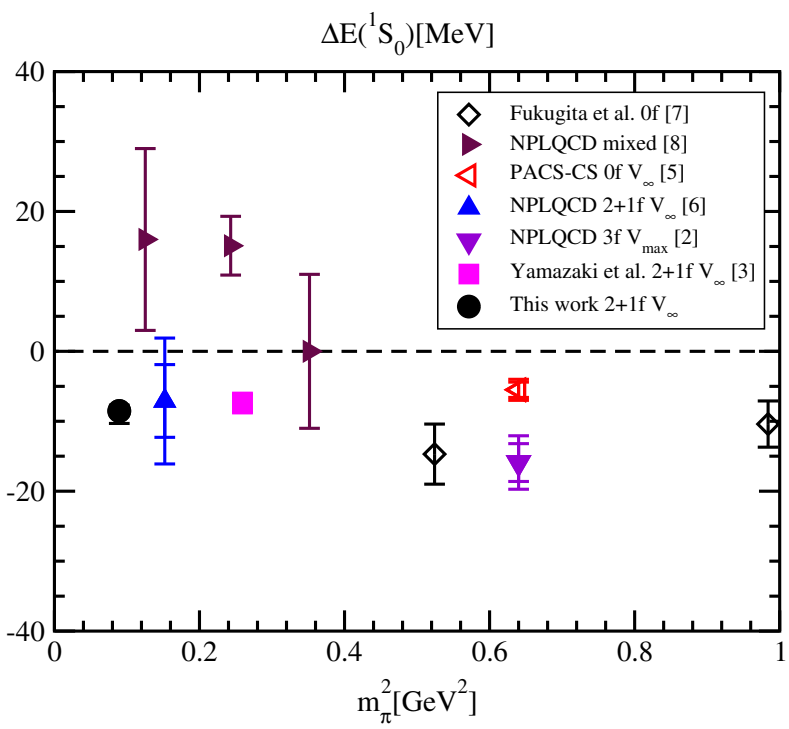

FIG. 18 (color online). Same as Fig. 17, but for the ${ }^{1} \mathrm{~S}_{0} N N$ channel.

calculations $[2,3,5,6]$ is a factor 5 to 10 times larger than the experimental value. Furthermore, we observe no tendency in the binding energy to approach the experimental value, at least over the pion mass range $m_{\pi}=0.3-0.51 \mathrm{GeV}$. For the ${ }^{1} \mathrm{~S}_{0}$ channel, the bound state found in the lattice calculations is absent in experiment. Furthermore, similarly to the ${ }^{3} \mathrm{~S}_{1}$ channel, the binding energy is almost flat in $m_{\pi}^{2}$ in the interval $m_{\pi}=0.30-0.51 \mathrm{GeV}$. It is not clear whether the bound state observed in the lattice calculation becomes unbound toward the physical $m_{\pi}$.

\section{CONCLUSION AND DISCUSSION}

We have extended our previous nuclei calculation in $2+1$ flavor QCD at $m_{\pi}=0.51 \mathrm{GeV}$ [3] to the lighter quark mass corresponding to $m_{\pi}=0.30 \mathrm{GeV}$ and $m_{N}=1.05 \mathrm{GeV}$. In order to suppress an exponential increase of statistical errors at smaller $m_{\pi}$, we have carried out a much larger number of measurements by a factor 12 and 5 for the case of the spatial extent of $4.3 \mathrm{fm}\left(48^{3}\right)$ and $5.8 \mathrm{fm}\left(64^{3}\right)$, respectively, compared to those for the $m_{\pi}=$ $0.51 \mathrm{GeV}$ case with the same volumes. We have found that in all channels we have studied, ${ }^{4} \mathrm{He},{ }^{3} \mathrm{He}$, and two-nucleon ${ }^{3} \mathrm{~S}_{1}$ and ${ }^{1} \mathrm{~S}_{0}$, the ground state is a bound state by investigating the volume dependence of energy shift $\Delta E_{L}$. The binding energies estimated for the infinite volume are as follows:

$$
-\Delta E_{\infty}= \begin{cases}47(7)\left(\begin{array}{l}
+20 \\
-11
\end{array}\right) \mathrm{MeV} & \text { for }{ }^{4} \mathrm{He} \\
21.7(1.2)\left({ }_{-1.6}^{+13}\right) \mathrm{MeV} & \text { for }{ }^{3} \mathrm{He} \\
14.5(0.7)\left(\begin{array}{l}
+2.4 \\
-0.8
\end{array}\right) \mathrm{MeV} & \text { for }{ }^{3} \mathrm{~S}_{1}, \\
8.5(0.7)\left({ }_{-0.5}^{+1.6}\right) \mathrm{MeV} & \text { for }{ }^{1} \mathrm{~S}_{0}\end{cases}
$$


These values differ little from those obtained at $m_{\pi}=$ $0.51 \mathrm{GeV}$ [3]. The largest relative difference occurs for the ${ }^{3} \mathrm{~S}_{1}$ channel, which is only a $1.9 \sigma$ effect if we use the total error adding the statistical and systematic ones by quadrature. Therefore, our conclusions at $m_{\pi}=0.30 \mathrm{GeV}$ are similar to those in Ref. [3] for $m_{\pi}=0.51 \mathrm{GeV}$ : the binding energy of the ${ }^{4} \mathrm{He}$ nucleus is comparable with the experimental value, while the ${ }^{3} \mathrm{He}$ nucleus and the deuteron are about 3 and 7 times larger than the experimental values, respectively, and a bound dineutron is observed in the ${ }^{1} \mathrm{~S}_{0}$ channel.

The differences we observe from experiment may arise from various sources, either computational or physical in origin. Statistical errors are fairly large in the calculations even for light nuclei. While the negative value of the energy shift is certain in all channels we looked at, better statistics and improved techniques will be welcome to better control the extraction of the energy shift for each volume and the infinite volume extrapolations.

The quark mass is heavier than experiment in all calculations to date. The binding in the ${ }^{1} \mathrm{~S}_{0}$ channel is shallower than the ${ }^{3} S_{1}$ so that the former bound state may become unbound as $m_{\pi}$ decreases toward the physical value. This can only be verified by calculations of the nuclear binding energy at smaller quark masses.

It is also possible that the finite lattice spacing effect is rather subtle. The short distance repulsion, in the language of nuclear potential, is possibly affected more by such effects than the long distance attraction, so that finite lattice spacing effects may push out the multinucleon wave function, and then the ground state would become a scattering state, for lattice spacings smaller than some value.
Another possible source of systematic error is excited state contaminations in the calculation. We have assumed that the nucleon and nucleus correlation functions are dominated by the ground state in the large $t$ region, where the plateau of $\Delta E_{L}^{\text {eff }}$ in Eq. (4) appears. While we have tuned the smearing parameter of the quark field to increase the overlap of the nucleus operator to its ground state, from the current data we cannot completely exclude the possibility that it is not sufficient to suppress the contaminations. To investigate the size of possible contaminations, we might try analyses with the variational method [30] using correlation function matrices.

For now, however, we think that a calculation at the physical point, keeping the lattice spacing, is the next step.

\section{ACKNOWLEDGMENTS}

Numerical calculations for the present work have been carried out on the FX10 cluster system at Information Technology Center of the University of Tokyo, on the T2KTsukuba cluster system and HA-PACS system and COMA system at University of Tsukuba, and on $\mathrm{K}$ computer at RIKEN Advanced Institute for Computational Science. We thank the colleagues in the PACS-CS collaboration for helpful discussions and providing us the code used in this work. This work is supported in part by Grants-in-Aid for Scientific Research from the Ministry of Education, Culture, Sports, Science, and Technology (No. 22244018 and No. 25800138) and Grants-in-Aid of the Japanese Ministry for Scientific Research on Innovative Areas (No. 20105002, No. 21105501, and No. 23105708).
[1] T. Yamazaki, Y. Kuramashi, and A. Ukawa (PACS-CS Collaboration), Phys. Rev. D 81, 111504 (2010).

[2] S. R. Beane, E. Chang, S. D. Cohen, W. Detmold, H. W. Lin, T. C. Luu, K. Orginos, A. Parreño, M. J. Savage, and A. Walker-Loud, Phys. Rev. D 87, 034506 (2013).

[3] T. Yamazaki, K.-i. Ishikawa, Y. Kuramashi, and A. Ukawa, Phys. Rev. D 86, 074514 (2012).

[4] T. Inoue, S. Aoki, T. Doi, T. Hatsuda, Y. Ikeda, N. Ishii, K. Murano, H. Nemura, and K. Sasaki (HAL QCD Collaboration), Nucl. Phys. A881, 28 (2012).

[5] T. Yamazaki, Y. Kuramashi, and A. Ukawa, Phys. Rev. D 84, 054506 (2011).

[6] S. R. Beane, E. Chang, W. Detmold, H. W. Lin, T. C. Luu, K. Orginos, A. Parreño, M. J. Savage, A. Torok, and A. Walker-Loud et al. (NPLQCD Collaboration), Phys. Rev. D 85, 054511 (2012).

[7] M. Fukugita, Y. Kuramashi, M. Okawa, H. Mino, and A. Ukawa, Phys. Rev. D 52, 3003 (1995).

[8] S. R. Beane, P. F. Bedaque, K. Orginos, and M. J. Savage, Phys. Rev. Lett. 97, 012001 (2006).
[9] N. Ishii (HAL QCD Collaboration), Proc. Sci., CD12 (2013) 025.

[10] Y. Iwasaki, arXiv:1111.7054.

[11] S. Aoki et al. (CP-PACS/JLQCD Collaborations), Phys. Rev. D 73, 034501 (2006).

[12] S. Aoki et al. (PACS-CS Collaboration), Phys. Rev. D 81, 074503 (2010).

[13] S. Aoki et al. (PACS-CS Collaboration), Phys. Rev. D 79, 034503 (2009).

[14] M. Lüscher, J. High Energy Phys. 05 (2003) 052.

[15] M. Lüscher, Comput. Phys. Commun. 165, 199 (2005).

[16] M. Hasenbusch, Phys. Lett. B 519, 177 (2001).

[17] K.-I. Ishikawa et al. (PACS-CS Collaboration), Proc. Sci., LAT2006 (2006) 027 [arXiv:hep-lat/0610037].

[18] I. P. Omelyan, I. M. Mryglod, and R. Folk, Comput. Phys. Commun. 151, 272 (2003).

[19] T. Takaishi and P. de Forcrand, Phys. Rev. E 73, 036706 (2006).

[20] W. Detmold and M. J. Savage, Phys. Rev. D 82, 014511 (2010). 
[21] T. Doi and M. G. Endres, Comput. Phys. Commun. 184, 117 (2013).

[22] W. Detmold and K. Orginos, Phys. Rev. D 87, 114512 (2013).

[23] J. Günther, B. C. Tóth, and L. Varnhorst, Phys. Rev. D 87, 094513 (2013).

[24] M. Lüscher, Commun. Math. Phys. 105, 153 (1986).

[25] S. R. Beane, P. F. Bedaque, A. Parreno, and M. J. Savage, Phys. Lett. B 585, 106 (2004).
[26] S. Sasaki and T. Yamazaki, Phys. Rev. D 74, 114507 (2006).

[27] S. R. Beane, W. Detmold, and M. J. Savage, Phys. Rev. D 76, 074507 (2007).

[28] J. E. Beam, Phys. Rev. 158, 907 (1967).

[29] M. Bolsterli and E. Jezak, Phys. Rev. 135, B510 (1964).

[30] M. Lüscher and U. Wolff, Nucl. Phys. B339, 222 (1990). 